\title{
The Blackburne-Peel Index for Determining Patellar Height Is Affected by Tibial Slope
}

\author{
Alexander J. Mortensen, B.S., Todd C. Ludwig, M.D., Benjamin M. Adams, \\ Temitope F. Adeyemi, M.D., and Stephen K. Aoki, M.D.
}

\begin{abstract}
Purpose: To establish a quantitative relationship between the Blackburne-Peel index and posterior tibial slope in both skeletally mature and skeletally immature individuals and to evaluate the rate at which variation in tibial slope influences changes in patellar height categorization as normal, patella alta, and patella baja. Methods: A consecutive series of lateral knee radiographs were retrospectively reviewed. Radiographs were excluded for rotation, inadequate visible proximal tibia length, and obstructive hardware/pathology. Modified tibial slopes of $0^{\circ}, 5^{\circ}, 10^{\circ}$, and $15^{\circ}$ were projected anteriorly from the medial tibial plateau as described by Blackburne-Peel. The Blackburne-Peel index was determined at each modified tibial slope interval. Caton-Deschamps and Insall-Salvati indices also were measured for comparison. The rate of Blackburne-Peel index change with increase in posterior tibial slope was quantitatively analyzed. Results: Fifty skeletally mature and 50 skeletally immature radiographs were included. In the skeletally mature, Blackburne-Peel indices decreased on average by $0.037,0.044$, and 0.049 as posterior tibial slope increased from $0-5^{\circ}, 5-10^{\circ}$, and 10 $15^{\circ}$, respectively. In the skeletally immature, Blackburne-Peel indices decreased on average by $0.045,0.053$, and 0.059 as posterior tibial slope increased from $0-5^{\circ}, 5-10^{\circ}$, and $10-15^{\circ}$, respectively. Overall, 29 individuals with $0^{\circ}$ of tibial slope were categorized as patella alta by the Blackburne-Peel index, and only $16(55 \%)$ remained categorized as patella alta after increasing their posterior tibial slope to $15^{\circ}$. Conclusions: This study quantitatively demonstrates the relationship between posterior tibial slope and the Blackburne-Peel index. As expected, as posterior tibial slope increases, the Blackburne-Peel index decreases. While the change in the Blackburne-Peel index per $5^{\circ}$ change in tibial slope appears to be small, nearly half $(45 \%)$ of individuals categorized as patella alta with $0^{\circ}$ of tibial slope were categorized as normal when their posterior tibial slope was systematically increased from $0^{\circ}$ to $15^{\circ}$. When evaluating patellar height, it is important to understand how tibial slope affects the Blackburne-Peel Index measurement. Clinical Relevance: As posterior tibial slope increases, the numerator of the Blackburne-Peel ratio decreases, and vice versa. This relationship can lead to incorrect assessment of patellar height. Objectively placing individuals into patella alta and baja categories may influence patient care and decision making.
\end{abstract}

$\mathbf{P}$ atellar height is clinically relevant to several pathologic processes, including patellofemoral pain, chondromalacia, patellofemoral osteoarthritis, tibial tubercle apophysitis, patellar subluxation, and patellar dislocation. ${ }^{1-3}$ Patellar height typically is assessed on lateral-view radiographs and sagittal plane imaging, and it is categorized as normal, patella alta, or patella baja. Patella alta describes a high-riding

From the School of Medicine (A.J.M.); University of Utah (B.M.A.); and Department of Orthopaedic Surgery (T.C.L., T.F.A., S.K.A.), University of Utah, Salt Lake City, Utah, U.S.A.

The authors report the following potential conflicts of interest or sources of funding: S.K.A. reports personal fees from Stryker, outside the submitted work. Full ICMJE author disclosure forms are available for this article online, as supplementary material.

The work for this manuscript was performed at the University of Utah in Salt Lake City, Utah, U.S.A.

Received May 17, 2020; accepted September 24, 2020. patella, whereas patella baja describes a low-riding patella.

The Insall-Salvati index (ISI), Caton-Deschamps index (CDI), and Blackburne-Peel index (BPI) measure different anatomic relationships between the patella and proximal tibia and are among the most commonly employed methods of assessing patellar height $^{2}$ (Fig 1). The ISI is a ratio of the patellar tendon

Address correspondence to Stephen K. Aoki, M.D., Department of Orthopaedic Surgery, 590 Wakara Way, Salt Lake City, UT 84108. E-mail: stephen.aoki@hsc.utah.edu

(C) 2020 THE AUTHORS. Published by Elsevier Inc. on behalf of the Arthroscopy Association of North America. This is an open access article under the CC BY-NC-ND license (http://creativecommons.org/licenses/by-nc-nd/4.0/). 2666-061X/20769

https://doi.org/10.1016/j.asmr.2020.09.026 
length and the diagonal length of the patella. ${ }^{4}$ The CDI is a ratio of the distance between the anterosuperior angle of the tibia and the inferior patellar articular surface, and the length of the patellar articular surface. ${ }^{5,6}$ The BPI is a ratio of the distance between a line projected anteriorly off of the slope of the tibial plateau and the inferior patellar articular surface, and the length of the patellar articular surface. ${ }^{7}$

The BPI previously has been reported to have lower interobserver variability and better discrimination between patella alta and baja than either the ISI or CDI methods. ${ }^{8}$ In addition, Phillips et al. ${ }^{9}$ performed a review of all methods used to determine patellar height and reported BPI as 1 of the 2 most reliable techniques on plain radiographs. The anteriorly projected line in the BPI is a tangent line of 2 points on the tibial plateau, which is the same tangent used to determine tibial slope (TS). ${ }^{10}$ The relationship between TS and BPI is unique among the commonly used methods of determining patellar height. ${ }^{9}$

There is a substantial amount of natural anatomic variation in TS among individuals. ${ }^{1,12}$ Two previous studies assessing posterior TS, one using computed tomography (CT) and another using magnetic resonance imaging (MRI), both found a mean posterior TS of $11^{\circ}$, with ranges of $5-17^{\circ}$ and $5-15.5^{\circ}$, respectively. ${ }^{11,12}$ In a study measuring the TS on the medial tibial plateau of 61 cadaveric tibias, the mean posterior TS was $8.4^{\circ} \pm$ $3.7^{\circ} .{ }^{13}$ Given that the technique described by Blackburne-Peel for determining patellar height is referenced off of TS, it is important to understand how this naturally encountered variation in TS can affect the ratio of the BPI. ${ }^{7}$

The purpose of this study was to establish a quantitative relationship between the BPI and posterior TS in both skeletally mature and skeletally immature individuals and to evaluate the rate at which variation in TS influences changes in patellar height categorization as normal, patella alta, and patella baja.

\section{Methods}

Each author certifies that his or her institution approved the human protocol for this investigation that all investigations were conducted in conformity with ethical principles of research, and that informed consent for participation in the study was obtained. This study was performed under the University of Utah Institutional Review Board-approved protocol \#71733. Consecutive lateral knee radiographs between January 1, 2018, and March 5, 2018, from a single university health care system were reviewed retrospectively. All knee radiographs were obtained from the university digital radiograph database. Inclusion criteria included any lateral knee radiograph with $30^{\circ}$ to $70^{\circ}$ of knee flexion to meet flexion criteria described in previous literature. ${ }^{4,5,7,9}$ Exclusion criteria included excessive rotation, inadequate proximal tibia in view to determine a reliable tibial axis, or hardware/pathology involving the knee such that it influenced or obstructed any radiographic measurements of the proximal tibia or patella.

On lateral radiographs of the knee, the anatomic tibial axis was determined using the tibial proximal anatomical axis. ${ }^{10}$ This was accomplished by positioning one point slightly distal to the tibial tubercle and another point at the most distal aspect of the tibia in view; both points were equidistant from the anterior and posterior cortices. A line placed orthogonal to the tibial axis and projected anteriorly from the anterosuperior aspect of the medial tibial plateau was used to determine a modified posterior TS of $0^{\circ}$ (Fig 2). BPI measurements were made using the anterior projection of this modified posterior TS, in the same manner one would use the anterior projection of an actual TS to measure BPI. ${ }^{7}$ The anterosuperior aspect of the medial tibial plateau then served as the fulcrum to increase modified posterior TS by $5^{\circ}$ intervals from $0^{\circ}$ to $15^{\circ}$. The BPI was measured at each $5^{\circ}$ interval. In addition, the CDI and ISI were measured on each radiograph. Each patellar height index was then categorized as normal, patella alta, or patella baja according to ranges reported in the current literature (BPI: patella alta $>1.06$, patella infera $<0.54$; ISI: patella alta $>1.2$, patella infera $<0.8$; CDI: patella alta $>1.3$, patella infera $<0.6) .{ }^{4-7,9}$ Proximal tibia length within radiographic view also was measured and recorded for each radiograph.

\section{Statistical Analysis}

Two observers (A.J.M., B.M.A.), blinded to each other's results, separately recorded each radiographic measurement, and an average of the 2 measurements of each patellar height index was recorded in every case. After a period of greater than 6 months, 20 radiographs (10 skeletally mature and 10 skeletally immature) were chosen at random and each patellar height index was remeasured in every case. Intraclass correlation coefficients (ICCs) and their 95\% confidence intervals (CIs) were calculated using SPSS statistical package, version 25 (IBM Corp, Armonk, NY) based on average measures, mean-rating $(\mathrm{k}=2)$, absolute-agreement, and a 2-way mixed effects model to determine inter-rater and intra-rater reliability. The mean ISI, CDI, and BPI at $0^{\circ}, 5^{\circ}, 10^{\circ}$, and $15^{\circ}$ of posterior TS were determined for both the skeletally mature and skeletally immature groups. The mean change in BPI was determined between each $5^{\circ}$ interval of posterior TS for both groups. All statistical analysis was performed in Excel (Microsoft, Redmond, WA) and IBM SPSS Statistics 25 (IBM Corp). 

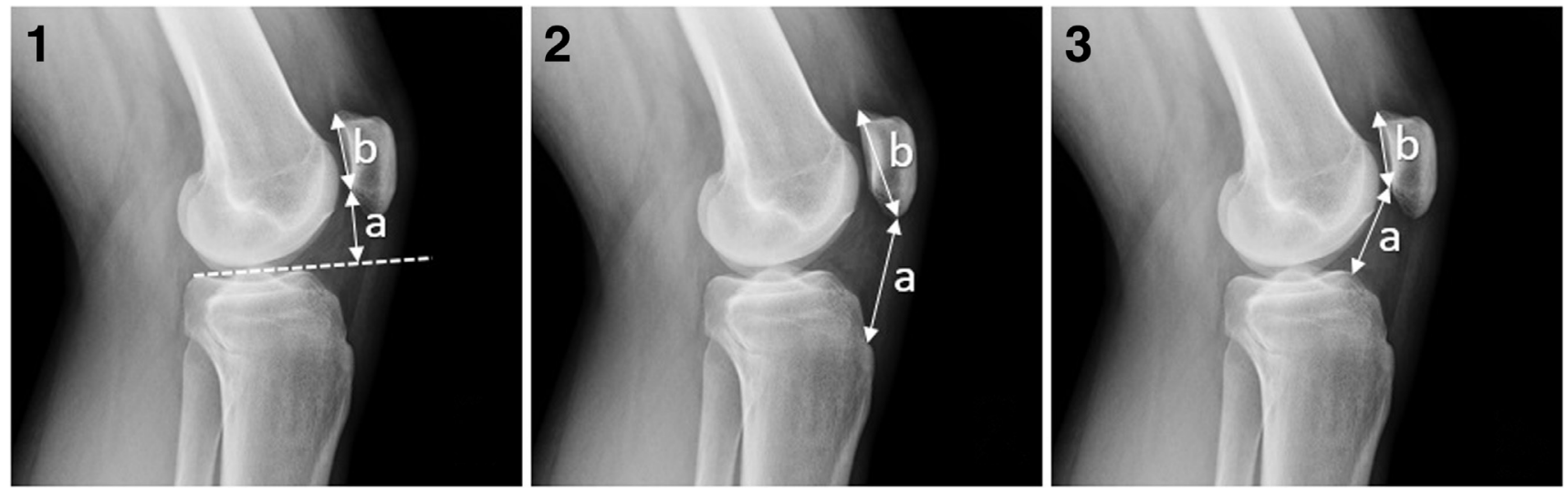

Fig 1. Lateral view of left knee. (1) BPI measurements: a) the perpendicular distance from a line projected anteriorly off of the tibial plateau slope to the inferior patellar articular surface and b) the length of the entire patellar articular surface. (2) ISI measurements: a) the distance from the tibial tubercle to the inferior pole of the patella and b) the diagonal length from the inferior pole of the patella to the superior patellar articular surface. (3) CDI measurements: a) the distance from the tip of the anterosuperior angle of the tibia to the inferior patellar articular surface and b) the length of the entire patellar articular surface. (BPI, Blackburne-Peel index; CDI, Caton-Deschamps index; ISI, Insall-Salvati index.)

\section{Results}

A total of 50 skeletally mature and 50 skeletally immature knees were included in the study. A total of 96 skeletally mature radiographs of individuals between the ages of 18 and 30 years old were reviewed. Eight were excluded for rotation, 1 was excluded for hardware, 15 were excluded for an inadequate proximal tibia length in view, 15 were excluded for inappropriate knee flexion, 6 were excluded for obstructive fracture, and 1 was excluded for obstructive heterotopic ossification. A total of 88 skeletally immature radiographs of individuals between the ages of 6 and 17 years old were reviewed. Five were excluded for rotation, 3 were excluded for hardware, 6 were excluded for an inadequate proximal tibia length in view, 19 were excluded for inappropriate knee flexion, and 5 were excluded for obstructive fracture. Demographic data for both groups including sex, age, height, weight, body mass index, and laterality is reported in Table 1 .

\section{Skeletally Mature}

Mean age of the skeletally mature group was $24 \pm 4.2$ years, ranging from 18 to 30 years old. The mean BPI at $0^{\circ}, 5^{\circ}, 10^{\circ}$, and $15^{\circ}$ was $0.94,0.91,0.86$, and 0.81 , respectively. As posterior TS was adjusted from 0 to $5^{\circ}$, 5 to $10^{\circ}$, and 10 to $15^{\circ}$, the mean change in BPI was $0.037,0.044$, and 0.049 , respectively. Mean ISI and CDI were 1.14 and 1.02 , respectively (Table 2 ). Patella alta was observed in $14 \%$ of $0^{\circ}$ BPI, $12 \%$ of $5^{\circ}$ BPI, $8 \%$ of $10^{\circ} \mathrm{BPI}, 6 \%$ of $15^{\circ} \mathrm{BPI}, 34 \%$ ISI, and $6 \%$ CDI. Patella baja was observed in $4 \%$ of $15^{\circ}$ BPI and $4 \%$ of ISI. Seven individuals with $0^{\circ}$ of TS were categorized as patella alta by BPI, and only $3(43 \%)$ remained categorized as patella alta after increasing their posterior TS to $15^{\circ}$ (Table 3). An average of $17.4 \mathrm{~cm}$ of proximal tibia was within the radiographic view for this group.

\section{Skeletally Immature}

Mean age of the skeletally immature group was $11.7 \pm 2.2$, ranging from 6 to 16 years old. The mean BPI at $0^{\circ}, 5^{\circ}, 10^{\circ}$, and $15^{\circ}$ were $1.04,1.00,0.94$, and 0.88 , respectively. As posterior TS was adjusted from 0 to $5^{\circ}, 5$ to $10^{\circ}$, and 10 to $15^{\circ}$, the mean change in BPI was $0.045,0.053$, and 0.059 , respectively. Mean ISI and CDI were 1.28 and 1.14 , respectively (Table 2). Patella alta was observed in $44 \%$ of $0^{\circ}$ BPI, $38 \%$ of $5^{\circ}$ BPI, $34 \%$ of $10^{\circ}$ BPI, $26 \%$ of $15^{\circ}$ BPI, $56 \%$ of ISI, and $16 \%$ of CDI. Patella baja was observed in $2 \%$ of $10^{\circ}$ BPI, $6 \%$ of $15^{\circ} \mathrm{BPI}$, and $2 \%$ of ISI. Twenty-two individuals with $0^{\circ}$ of TS were categorized as patella alta by BPI, and only $13(59 \%)$ remained categorized as patella alta after increasing their posterior TS to $15^{\circ}$ (Table 3). An average of $14.9 \mathrm{~cm}$ of proximal tibia was within the radiographic view for this group.

The inter-rater reliability ICCs for the skeletally mature group was 0.981 (95\% CI 0.966-0.989), 0.873 (95\% CI $0.773-0.929)$, and 0.866 (95\% CI $0.774-$ $0.914)$ for the ISI, CDI, and BPI, respectively. The interrater reliability ICCs for the skeletally immature group were 0.970 (95\% CI $0.947-0.983), 0.920 \quad(95 \%$ CI $0.765-0.965$ ), and 0.897 (95\% CI 0.799-0.940) for the ISI, CDI, and BPI, respectively. Reader 1 intra-rater reliability ICCs were 0.954 (95\% CI $0.605-0.988)$, 0.959 (95\% CI 0.898-0.984), and 0.914 (95\% CI 0.865 0.945 ) for the ISI, CDI, and BPI, respectively. Reader 2 intra-rater reliability ICCs were 0.993 (95\% CI 0.983 $0.997), 0.941$ (95\% CI 0.841-0.977), and 0.917 (95\% CI 0.607-0.968) for the ISI, CDI, and BPI, respectively. 


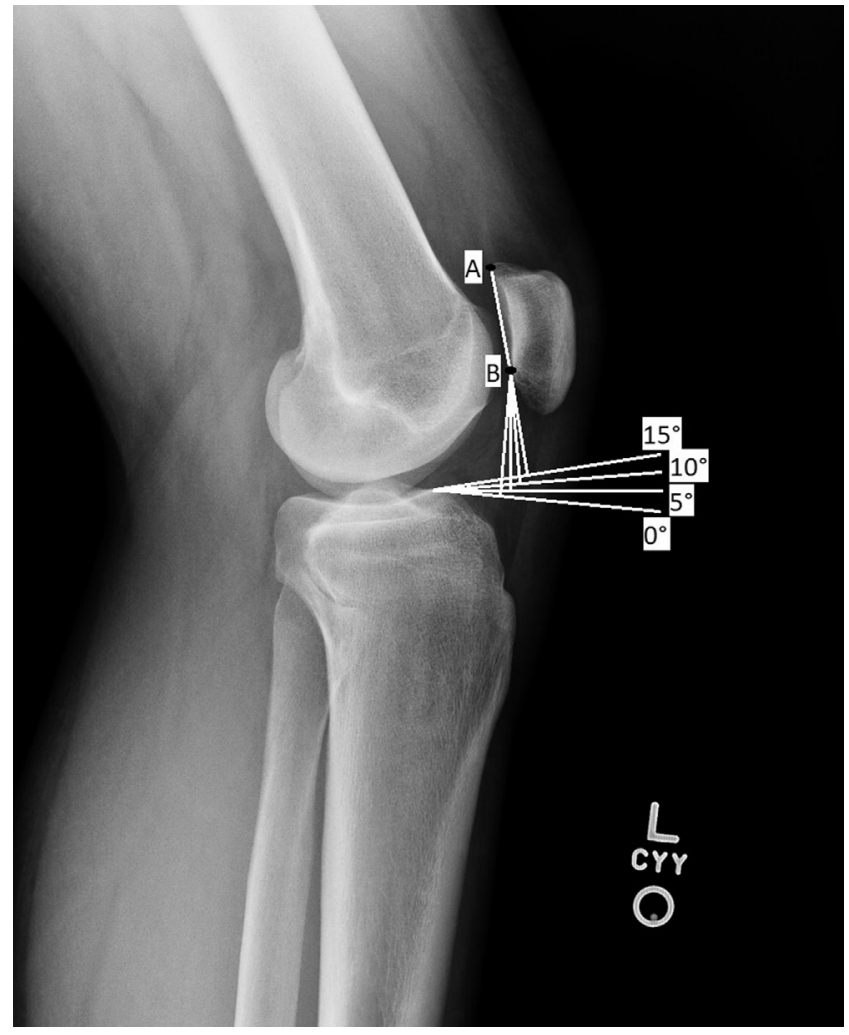

Fig 2. Lateral view of a left skeletally mature knee with superimposed BPI measurements. Anterior projections of the anterior medial tibial plateau are labeled at $0^{\circ}, 5^{\circ}, 10^{\circ}$, and $15^{\circ}$ of modified posterior TS. Point A to point $\mathrm{B}$ measures the patellar articular surface, and is the denominator of the BPI. Lines from point B measure from the inferior aspect of the patellar articular surface to the anterior projection of tibial slope. This measurement, the numerator of BPI, is directly affected by tibial slope. (BPI, Blackburne-Peel index.)

All ICCs fell within the range of "good" (0.75-0.90) and "excellent" (>0.90).

\section{Discussion}

This study demonstrated the quantitative relationship between posterior TS and BPI in both skeletally mature and skeletally immature individuals. In skeletally mature individuals, BPI decreased on average by 0.043 for every $5^{\circ}$ increase in posterior TS. In skeletally immature individuals, BPI decreased on average by 0.052 for every $5^{\circ}$ increase in posterior TS. The BPI is one of a few commonly used methods of determining patellar height. One of the 2 components of the BPI, the numerator, measures an orthogonal line from the anterior projection of the TS to the inferior aspect of the patellar articular surface. An anticipated limitation with the BPI is that variation in posterior TS among individuals can influence the numerator component of the BPI measurement.

There is a significant amount of posterior TS variation among individuals. A cadaveric study by de Boer
Table 1. Cohort Demographics

\begin{tabular}{|c|c|c|}
\hline Variable & $\begin{array}{c}\text { Skeletally Mature } \\
(\mathrm{N}=20)\end{array}$ & $\begin{array}{l}\text { Skeletally Immature } \\
(\mathrm{N}=20)\end{array}$ \\
\hline$\overline{\text { Age, mean (SD) }}$ & $24.0(4.2)$ & $11.7(2.2)$ \\
\hline Range & $(18-30)$ & $(6-16)$ \\
\hline Female, n (\%) & $24(48)$ & $26(52)$ \\
\hline Male, n (\%) & $26(52)$ & $24(48)$ \\
\hline $\begin{array}{l}\text { Height* }(\mathrm{cm}), \\
\text { mean }(\mathrm{SD})\end{array}$ & $173.5(12.80)$ & $156.9(14.6)$ \\
\hline Range & $(149.9-208.3)$ & (119.4-182.9) \\
\hline $\begin{array}{l}\text { Weight, kg, } \\
\text { mean (SD) }\end{array}$ & $84.4(22.0)$ & $54.2(20.7)$ \\
\hline Range & $(51.7-147.1)$ & $(20.6-105.2)$ \\
\hline $\begin{array}{l}\text { BMI, }{ }^{*} \text { mean } \\
\quad(\mathrm{SD})\end{array}$ & $28.0(6.8)$ & $21.3(6.1)$ \\
\hline Range & $(18.4-49.3)$ & $(14.4-37.3)$ \\
\hline Left, n (\%) & $29(58)$ & $20(40)$ \\
\hline Right, n (\%) & $21(42)$ & $30(60)$ \\
\hline
\end{tabular}

BMI, body mass index; SD, standard deviation.

*One patient was missing a height and BMI measurement in the skeletally immature group.

et al., ${ }^{13}$ showed the mean posterior TS to be $8.4^{\circ}$, with 1 standard deviation falling between $4.7^{\circ}$ and $12.1^{\circ}$. Matsuda et al. ${ }^{12}$ showed posterior TS measured from the medial tibial plateau of 30 individuals with normal knees to have a range of $5^{\circ}$ and $15.5^{\circ}$. Ho et al. ${ }^{11}$ measured posterior TS on normal knees of 100 individuals using 3-dimensional computed tomography and determined a 2 standard deviation range of $5^{\circ}$ to $17^{\circ}$. The current study assessed patellar height with changes in posterior TS at $5^{\circ}$ intervals between $0^{\circ}$ and $15^{\circ}$, which is approximately the same range described in the aforementioned studies. Due to the relatively large variation in "normal" posterior TS, it is important to understand the relationship between posterior TS and BPI, and how this relationship can affect values of patellar height obtained when using BPI. The normal range of BPI is 0.54-1.06, and the results of our study show the magnitude of change in BPI per $5^{\circ}$ change in posterior TS is relatively small compared with the normal range of BPI. In addition, in our study the mean BPI values with $0^{\circ}, 5^{\circ}, 10^{\circ}$, and $15^{\circ}$ of posterior TS are all within the normal BPI range for both the skeletally mature and immature groups. These findings might suggest variable posterior TS does not substantially influence BPI patellar height categorization. However, in the skeletally mature group of this study, an $8 \%$ increase in patella alta was observed with $0^{\circ}$ posterior TS compared with $15^{\circ}$ posterior TS. In the skeletally immature group of this study, an $18 \%$ increase in patella alta was observed with $0^{\circ}$ posterior TS compared with $15^{\circ}$ posterior TS. Furthermore, in this study 29 individuals with $0^{\circ}$ of TS were categorized as patella alta by the BPI; however, only $55 \%$ of those remained categorized as patella alta after increasing their posterior TS to $15^{\circ}$. These findings suggest that BPI values 
Table 2. Patellar Height Indices of the Skeletally Mature and Immature Groups

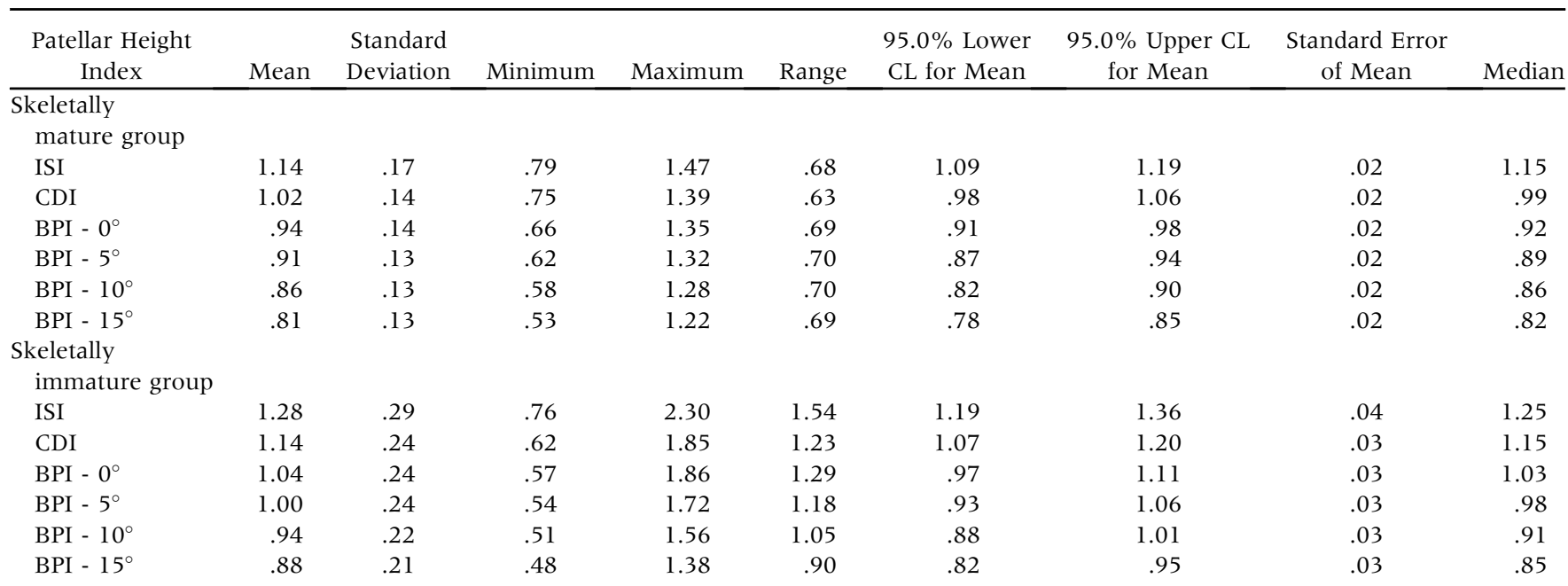

NOTE. Shown are ISI, CDI, and BPI at $0^{\circ}, 5^{\circ}, 10^{\circ}$, and $15^{\circ}$. Normal ranges for each patellar height index: BPI: patella alta $>1.06$, patella infera $<0.54$; ISI: patella alta $>1.2$, patella infera $<0.8$; CDI: patella alta $>1.3$, patella infera $<0.6$.

BPI, Blackburne-Peel index; CDI, Caton-Deschamps index; CL, confidence Limit; ISI, Insall-Salvati index.

should be interpreted with caution when evaluating patellar height in individuals with TS at the extremes of the normal range.

The majority of research conducted on the relationship between posterior TS and patellar height has focused on high tibial osteotomy (HTO) and total knee arthroplasty. In a prospective study of patients undergoing medial opening wedge HTO, LaPrade et al. ${ }^{14}$ showed a significant decrease in average BPI from 0.91 to 0.80 while also showing a significant increase in average posterior TS from $9.0^{\circ}$ to $11.9^{\circ}$. However, their study did not demonstrate a quantitative relationship between BPI and posterior TS. Furthermore, Kesmezacar et al., ${ }^{15}$ in a study of 85 patients who underwent valgus HTO, made the observation that BPI is an unreliable measurement of patellar height because of the fact that posterior TS is altered in the surgery. El-Azab et al., ${ }^{16}$ using CDI to assess patellar height, showed no correlation between change in posterior TS and patellar height following both closing wedge and opening wedge HTO surgeries. However, the authors only reported on CDI in relation to posterior TS and did not report on any potential correlation between posterior TS and BPI.

The accuracy in measuring TS can be influenced in a number of ways. Rotation of the lower extremity is common in lateral knee radiographs and leads to variable error in assessment of TS. A cadaveric study by Kessler et al. ${ }^{17}$ showed error in the measurement of TS reached $14^{\circ}$ as the tibia was internally rotated to $30^{\circ}$. Rotation also can influence the profile of the tibia, femur, and patella seen on lateral knee radiographs, making evaluation for rotation important before measuring patellar height with any method. Accuracy of TS measurements also may be influenced by incorrect assessment of the tibial axis. ${ }^{18}$ Faschingbauer

Table 3. Classification of Patella Height by Indices for Skeletally Mature and Immature Groups

\begin{tabular}{|c|c|c|c|c|c|c|}
\hline Patellar Height Category & ISI & $\mathrm{CDI}$ & $\mathrm{BPI}-0^{\circ}$ & BPI $-5^{\circ}$ & BPI - $10^{\circ}$ & $\mathrm{BPI}-15^{\circ}$ \\
\hline \multicolumn{7}{|l|}{ Skeletally mature group } \\
\hline Patella alta, n (\%) & $17(34 \%)$ & $3(6 \%)$ & $7(14 \%)$ & $6(12 \%)$ & $4(8 \%)$ & $3(6 \%)$ \\
\hline Patella baja, n (\%) & $2(4 \%)$ & 0 & 0 & 0 & 0 & $2(4 \%)$ \\
\hline \multicolumn{7}{|l|}{ Skeletally immature group } \\
\hline Patella alta, n (\%) & $28(56 \%)$ & $8(16 \%)$ & $22(44 \%)$ & $19(38 \%)$ & $17(34 \%)$ & $13(26 \%)$ \\
\hline Patella baja, n (\%) & $1(2 \%)$ & 0 & 0 & 0 & $1(2 \%)$ & $3(6 \%)$ \\
\hline
\end{tabular}

NOTE. Shown are ISI, CDI, and BPI at $0^{\circ}, 5^{\circ}, 10^{\circ}$, and $15^{\circ}$.

BPI, Blackburne-Peel index; CDI, Caton-Deschamps index; ISI, Insall-Salvati index.

Normal ranges for each patellar height index. BPI: patella alta $>1.06$, patella baja $<0.54$; ISI: patella alta $>1.2$, patella baja $<0.8$; CDI: patella alta $>1.3$, patella baja $<0.6$. 
et al. ${ }^{19}$ showed TS is most accurately measured with long lateral knee radiographs (defined as $16-20 \mathrm{~cm}$ of proximal tibia in view) and short view radiographs may overestimate TS by approximately $3^{\circ}$. The average length of the tibia in view in the current study was 17.4 and $14.9 \mathrm{~cm}$ in the skeletally mature and skeletally immature groups, respectively. Tibial length of the skeletally immature is shorter on average; thus, we feel that a slightly shorter length of tibia was reasonable to accurately determine the tibial axis in this group.

This study demonstrated mean BPI values in the skeletally immature group were larger at each degree of posterior TS when compared with the skeletally mature group. A major contribution accounting for this difference between the 2 groups is the disparity in patellar size. A relatively smaller patella in the skeletally immature group is associated with a smaller patellar articular surface, resulting in a relative increase to the BPI. In addition, a relatively smaller patella causes the point of the inferior aspect of the patellar articular surface to move proximally. Thus, the numerator would be expected to increase, again influencing an increase in the BPI of skeletally immature individuals. A recent study assessing the BPI and CDI in children 6 to 12 years old determined the average median values to be 1.07 and 1.22 for BPI and CDI, respectively. ${ }^{20}$ These findings are similar to the current study in that they show patellar height values in the skeletally immature are larger than normal values of patellar height reported in skeletally mature adults.

Multiple studies have shown significant anatomical differences between the slopes of the medial tibial plateau and lateral tibial plateau, and distinguishing between the medial and lateral tibial plateaus on lateral knee radiographs can be particularly challenging. Making an incorrect assessment of the medial plateau can lead to significant inaccuracies in the measurement of TS. ${ }^{12,21,22}$ A strength of the measurement method used in the current study is that it removed any error attributed to wrong discrimination between lateral and medial tibial plateaus. MRI and CT imaging modalities provide a clear distinction between medial and lateral tibial plateaus as they are viewed on separate images. Using a novel technique of determining the tibial axis with a conventional knee MRI, Hudek et al. ${ }^{23}$ demonstrated the mean TS was $4.8^{\circ}$. This was $3.4^{\circ}$ less than what they found in the same patients on lateral radiographs. In their observation, they noted it can be particularly difficult to distinguish between the lateral and medial tibial plateaus on lateral radiographs. In addition, they found 21 of 100 patients had a greater than $5^{\circ}$ difference in posterior TS between medial and lateral tibial plateaus. In contrast to the mean posterior TS findings by Hudek et al., Matsuda et al. ${ }^{12}$ evaluated MRIs that provided the entire view of the tibia to determine the longitudinal tibial axis and reported a mean posterior TS of $10.7^{\circ}$ using the medial tibial plateau in normal knees. Matsuda et al.'s findings are more consistent with previous radiographic and CT studies assessing mean posterior TS. ${ }^{11,24}$ While MRI and 3-dimensional CT are effective at distinguishing between the medial and lateral tibial plateaus and may be used to determine TS, they are costly and timeconsuming. Lateral knee radiographs remain the most common clinically used imaging modality for assessing patellar height.

\section{Limitations}

This study has some limitations. First, actual TS were not used in this study; thus, the study is theoretical in design. Second, this study was not designed to evaluate the clinical relevance of the quantitative relationship between TS and BPI. Third, the relationship between TS and BPI was only assessed using plain radiographs, no other imaging modalities were used. However, this is likely more practical, as patellar height is typically assessed clinically using plain radiographs. Furthermore, the original description of the BPI was described using radiographs, not 3-dimensional imaging. Finally, our study included assessment of 100 consecutive radiographs, and a larger sample size could potentially improve the validity of our findings.

\section{Conclusions}

This study quantitatively demonstrates the relationship between posterior TS and the BPI. As expected, as posterior TS increases, the BPI decreases. While the change in the BPI per $5^{\circ}$ change in TS appears to be small, nearly one half $(45 \%)$ of individuals categorized as patella alta with $0^{\circ}$ of TS were categorized as normal when their posterior TS was systematically increased from $0^{\circ}$ to $15^{\circ}$. When evaluating patellar height, it is important to understand how TS affects the BPI measurement.

\section{References}

1. Lancourt JE, Cristini JA. Patella alta and patella infera. Their etiological role in patellar dislocation, chondromalacia, and apophysitis of the tibial tubercle. J Bone Joint Surg Am 1975;57:1112-1115.

2. Biedert RM, Tscholl PM. Patella alta: A comprehensive review of current knowledge. Am J Orthop (Belle Mead NJ) 2017;46:290-300.

3. Askenberger M, Janarv PM, Finnbogason T, Arendt EA. Morphology and anatomic patellar instability risk factors in first-time traumatic lateral patellar dislocations: A prospective magnetic resonance imaging study in skeletally immature children. Am J Sports Med 2017;45:50-58.

4. Insall J, Salvati E. Patella position in the normal knee joint. Radiology 1971;101:101-104.

5. Caton J. Method of measuring the height of the patella. Acta Orthop Belg 1989;55:385-386. 
6. Caton J, Deschamps G, Chambat P, Lerat JL, Dejour H. Patella infera. Apropos of 128 cases. Rev Chir Orthop Reparatrice Appar Mot 1982;68:317-325 [in French].

7. Blackburne JS, Peel TE. A new method of measuring patellar height. J Bone Joint Surg Br 1977;59:241-242.

8. Seil R, Müller B, Georg T, Kohn D, Rupp S. Reliability and interobserver variability in radiological patellar height ratios. Knee Surg Sports Traumatol Arthrosc 2000;8:231-236.

9. Phillips CL, Silver DA, Schranz PJ, Mandalia V. The measurement of patellar height: A review of the methods of imaging. J Bone Joint Surg Br 2010;92:1045-1053.

10. Brazier J, Migaud H, Gougeon F, Cotten A, Fontaine C, Duquennoy A. Evaluation of methods for radiographic measurement of the tibial slope. A study of 83 healthy knees. Rev Chir Orthop Reparatrice Appar Mot 1996;82: 195-200 [in French].

11. Ho JPY, Merican AM, Hashim MS, Abbas AA, Chan CK, Mohamad JA. Three-dimensional computed tomography analysis of the posterior tibial slope in 100 knees. J Arthroplasty 2017;32:3176-3183.

12. Matsuda S, Miura H, Nagamine R, et al. Posterior tibial slope in the normal and varus knee. Am J Knee Surg 1999;12:165-168.

13. de Boer JJ, Blankevoort L, Kingma I, Vorster W. In vitro study of inter-individual variation in posterior slope in the knee joint. Clin Biomech (Bristol, Avon) 2009;24:488-492.

14. LaPrade RF, Oro FB, Ziegler CG, Wijdicks CA, Walsh MP. Patellar height and tibial slope after opening-wedge proximal tibial osteotomy: A prospective study. Am J Sports Med 2010;38:160-170.

15. Kesmezacar H, Erginer R, Ogut T, Seyahi A, Babacan M, Tenekecioglu Y. Evaluation of patellar height and measurement methods after valgus high tibial osteotomy. Knee Surg Sports Traumatol Arthrosc 2005;13:539-544.
16. El-Azab H, Glabgly P, Paul J, Imhoff AB, Hinterwimmer S. Patellar height and posterior tibial slope after open- and closed-wedge high tibial osteotomy: A radiological study on 100 patients. Am J Sports Med 2010;8:323-329.

17. Kessler MA, Burkart A, Martinek V, Beer A, Imhoff AB. Development of a 3-dimensional method to determine the tibial slope with multislice-CT. Z Orthop Ihre Grenzgeb 2003;141:143-147.

18. Ahmad R, Patel A, Mandalia V, Toms A. Posterior tibial slope: Effect on, and interaction with, knee kinematics. JBJS Rev 2016;4:e31-e36.

19. Faschingbauer M, Sgroi M, Juchems M, Reichel H, Kappe T. Can the tibial slope be measured on lateral knee radiographs? Knee Surg Sports Traumatol Arthrosc 2014;22: 3163-3167.

20. Vergara-Amador E, Davalos Herrera D, Guevara OA. Normal values of 3 methods to determine patellar height in children from 6 to 12 years. Rev Esp Cir Ortop Traumatol 2018;62:442-447.

21. Chiu KY, Zhang SD, Zhang GH. Posterior slope of tibial plateau in Chinese. J Arthroplasty 2000;15:224-227.

22. Han HS, Chang CB, Seong SC, Lee S, Lee MC. Evaluation of anatomic references for tibial sagittal alignment in total knee arthroplasty. Knee Surg Sports Traumatol Arthrosc 2008;16:373-377.

23. Hudek R, Schmutz S, Regenfelder F, Fuchs B, Koch PP. Novel measurement technique of the tibial slope on conventional MRI. Clin Orthop Relat Res 2009;467: 2066-2072.

24. Yoo JH, Chang CB, Shin KS, seong SC, Kim TK. Anatomical references to assess the posterior tibial slope in total knee arthroplasty: A comparison of 5 anatomical axes. J Arthroplasty 2008;23:586-592. 